\title{
Pareto-Verbesserungen in der Sozialversicherung durch Selbstselektion ihrer Mitglieder
}

von

\author{
Michael Breuer ${ }^{1}$
}

\begin{abstract}
In der Sozialversicherung gelten Risikoselektionen als unerwünscht, weil sie die Gefahr mit sich bringen, dass der einheitliche Risikopool in der Sozialversicherung aufgespalten und die sozialpolitisch gewünschten Umverteilungsströme zwischen den Versicherten reduziert werden. Der Beitrag zeigt am Beispiel der sozialen Krankenversicherung in der Schweiz und der Bundesrepublik Deutschland auf, dass die Selbstselektion von Versicherten in der Sozialversicherung produktiv, das heisst wohlfahrtssteigernd eingesetzt werden kann, ohne den ,solidarischen ' Charakter der Sozialversicherung in Frage zu stellen. Konkret wird vorgeschlagen, den Versicherten innerhalb eines durch einen Risikoausgleich gestützten Wettbewerbs zwischen den Krankenkassen eine grössere Wahlfreiheit über die Höhe des Versicherungsschutzes einzuräumen. Für Versicherte mit niedrigem Risiko oder hohem Einkommen kann es sinnvoll sein, ihren Versicherungsschutz innerhalb der Sozialversicherung zu reduzieren, obwohl sie dann aufgrund des konstanten Transfers an andere Versicherte der sozialen Krankenversicherung einen höheren Preis pro Einheit Sozialversicherungsschutz zu zahlen haben.
\end{abstract}

JEL classification:

$\mathrm{H} 55, \mathrm{H} 230$

1) Sozialökonomisches Seminar, Universität Zürich, Blümlisalpstr. 10, CH-8006 Zürich, Switzerland. InternetAdresse: http//:www.unizh.ch/sozoec. E-mail: mbreuer@sozoec.unizh.ch. Der Autor dankt Jörg Wild und Prof. Peter Zweifel, beide Universität Zürich, für wertvolle Hinweise und Anregungen. 


\section{Einleitung}

Die Selbstselektion von Versicherungsnehmern ist in der Literatur der Versicherungsökonomik seit langem ein Thema. Am bekanntesten dürfte das Rothschild-Stiglitz-Modell sein, in dem die Selbstselektion von Versicherten genutzt wird, um die nachteiligen Auswirkungen von Informationsasymmetrien auf einem Versicherungsmarkt wenigstens zum Teil zu überwinden und evtl. trotz der unvollkommenen Information der Versicherer zu einem Gleichgewicht auf dem privaten Versicherungsmarkt zu gelangen, in dem kein Anbieter einen Verlust realisiert und kein Versicherter noch einen Anreiz hat, das Angebot eines anderen Versicherers anzunehmen. ${ }^{1}$ Unter den Annahmen, die Rothschild/Stiglitz (1976) zum Verhalten miteinander im Wettbewerb stehender Versicherungsunternehmen machen ${ }^{2}$, kann es in einem wettbewerblichen Versicherungsmarkt nicht zum einem Gleichgewicht kommen, in dem die guten und die schlechten Risiken den gleichen Versicherungsvertrag wählen. Einzig denkbar ist ein Separierungsgleichgewicht, das sich durch eine Teildeckung für die guten und einen vollen Versicherungsschutz für die schlechten Risiken auszeichnet. Die Autoren weisen jedoch nach, dass die Existenz eines solchen Gleichgewichtes auf dem privaten Versicherungsmarkt keinesfalls garantiert ist. Es kann somit in ihrem Modell auch zu völligem Marktversagen und zum Zusammenbruch des Versicherungsmarktes kommen.

Nicht zuletzt solche Marktunvollkommenheiten werden als Begründungen für die Einführung einer (staatlichen) Pflichtversicherung angeführt, in der alle Versicherten, gleich welches Versicherungsrisiko sie bergen, zwangsweise in einen einzigen Versicherungspool zusammengeführt werden. ${ }^{3}$ Die Idee eines einheitlichen Versichertenpools liegt letztlich auch der Sozialversicherung zugrunde, wobei die konkrete Ausgestaltung und Zusammensetzung des Pools sowie dessen Finanzierungsregeln nicht nur von Land zu Land, sondern auch von einem Sozialversicherungszweig zum anderen variiert. Wenn wir den Sozialversicherungszweig der Krankenversicherung herausgreifen, dann bestehen z.B. zwischen Deutschland und der Schweiz deutliche Unterschiede. So können sich in Deutschland die Bezieher hoher Einkommen der Sozialversicherungspflicht in der Gesetzlichen Krankenversicherung (GKV) vollkommen entziehen. Die Beiträge zur GKV werden einkommensabhängig erhoben (bis zur Beitragsbemessungsgrenze, die in der GKV zugleich auch die Versicherungspflichtgrenze darstellt) um, nach dem Willen der sozialpolitischen Gestalter zugleich einen , sozialen Ausgleich`zwischen den Beziehern hoher und niedrigerer Einkommen innerhalb der GKV

1) Das Rothschild-Stiglitz-Gleichgewicht stellt also ein Second-Best-Optimum dar.

2) Durch andere Verhaltensannahmen werden neue Gleichgewichtssituationen denkbar. So kann es nach Wilson (1977) durchaus auch zu Pooling-Gleichgewichten kommen, wenn Versicherer, die potentiell versucht sein könnten, mit ihren Versicherungspolicen nur gute Risiken anzusprechen, antizipieren, dass dadurch die bislang auf dem Markt befindlichen Versicherungsverträge nicht mehr aufrecht erhalten werden können. Letztlich würde der , Angreifer damit auch die schlechten Risiken aufzunehmen haben. Vgl. für eine Zusammenfassung möglicher Versicherungsmarktgleichgewichte neueren Datums Windisch (1998). Strassl (1988, 166) begründet, warum in Konkurrenzsituationen die Rothschild-Stiglitz-Annahmen plausibler erscheinen.

3) Vgl. für eine formale Darstellung z.B. Strassl (1988, 131ff.). 
zu schaffen. In der Schweiz dagegen müssen alle Einwohner einen Krankenversicherungsschutz bei einer Versicherungsgesellschaft ihrer Wahl nachweisen, wobei die einzelnen Gesellschaften einen gesetzlich definierten Grundversorgungstarif anbieten müssen, für den sie keine risikoabhängigen Prämien erheben und keine Versicherungsschutz suchenden Individuen abweisen dürfen. Der , soziale Ausgleich“ findet innerhalb der sozialen Krankenversicherung der Schweiz daher nur zwischen den guten und den schlechten Risiken statt. ${ }^{1}$ Einkommensunterschiede werden nicht explizit berücksichtigt. Dafür ist es aber auch niemandem möglich, sich der Teilnahme am Risikoausgleich im Rahmen des Grundversorgungstarifs zu entziehen, wie dies in Deutschland Praxis ist.

Eine Einheitsversicherung in Verbindung mit einer Zwangsmitgliedschaft kann nicht nur verhindern, dass sich einzelne dem ,sozialen Ausgleich“ entziehen, sondern ermöglicht obendrein auch noch eine kostengünstige Akquisition von Versicherten. Jedoch entstehen in einer Einheitsversicherung neue ökonomische Probleme: Zum einen ist durch die aufgrund des fehlenden Wettbewerbs möglicherweise ineffiziente Mittelverwendung innerhalb der Versicherung die kostengünstige Bereitstellung des Versicherungsschutzes wieder in Frage gestellt. Zum anderen stellt sich die Frage, wie es zu gewährleisten ist, dass sich der Umfang des Sozialversicherungsschutzes an den Präferenzen der Versicherten orientiert, wenn sie, statt der einfach zu handhabenden marktlichen ExitOption, auf die (politische) Voice-Option verwiesen sind, die gerade in Anbetracht des Kollektivgutproblems, wie es zwischen den Sozialversicherten besteht, kaum eine wirkungsvolle Alternative darstellt.

Um aber Anreize für eine kostengünstige Versorgung der Versicherten mit den Leistungen der Krankenversicherung zu schaffen, hat man sich inzwischen in beiden Ländern für einen regulierten Wettbewerb zwischen den Krankenkassen entschieden, der durch einen Risikoausgleich ${ }^{2}$ zwischen den Anbietern flankiert wird. Mit dem Risikoausgleich soll der in der Praxis unvermeidliche Tradeoff zwischen der Vollständigkeit des Risikopools und der Chance, mittels Wettbewerb zu einer kostengünstigeren Leistungserstellung zu kommen, möglichst gering gehalten werden. Der Risikoausgleich soll gewährleisten, dass sich die allokativ gewünschten Grössen im Beitrag, bzw. Beitragssatz der Kassen niederschlagen. Der Beitrag, den die einzelnen Kassen verlangen, soll die Wirtschaftlichkeit der Leistungserstellung widerspiegeln und nicht von der in den einzelnen Kassen vorzufindenden Risikostruktur (und Einkommensstruktur bei den deutschen Kassen) beeinflusst sein. Damit soll zugleich ausgeschlossen werden, dass sich die Versicherten stets für die Krankenkasse mit der gerade günstigeren Einkommens- und Risikostruktur entscheiden und durch ihre Wanderungsbewegungen, die letztlich nur dem Ziel dienen, sich im Verteilungskampf eine günstigere

1) Allerdings werden in der Schweiz aus allgemeinen Haushaltsmitteln Prämienverbilligungen in der Grundversicherung subventioniert, wodurch sich unter Einbezug der Steuern auch zwischen den Beziehern hoher und niedriger Einkommen über die Krankenversicherung ein gewisser Ausgleich ergibt.

2) In der deutschen Literatur ist der Begriff Risikostrukturausgleich vorherrschend, während in der Schweiz der kürzere Begriff Risikoausgleich bevorzugt wird. Dieser findet auch im folgenden Verwendung. 
Position zu verschaffen, zu einer Destabilisierung des sozialen Krankenversicherungssystems beitragen (Breuer 1998, Kapitel II). Auch wenn man sich in der Literatur noch nicht einig ist, ob der Risikoausgleich eine permanente Einrichtung bleiben muss, oder ob er nach einer gewissen Angleichung der Risikostruktur zwischen den Kassen wieder fallengelassen werden kann ${ }^{1}$, wird er doch als der entscheidende Schritt für eine Wettbewerbsordnung in einer Krankenversicherung gewertet (Leber 1992), in der es den Kassen nicht erlaubt ist, individuell risikoabhängige Beiträge zu erheben. Risikoselektionen gelten in einem solchen Krankenversicherungssystem als unerwünscht, weil sie letztlich die Bildung eines Pools verhindern. Statt dessen besteht die Gefahr, dass nur Kosten von einem Versicherer zum nächsten weitergeschoben werden und ineffiziente Anbieter im Wettbewerb überleben, die es geschafft haben, sich auf Kosten ihrer Konkurrenten von den schlechten Risiken frei zu halten (van de Ven 1992, 24).

Verhindert man jegliche Selbstselektion der Sozialversicherten, bleibt aber das Problem, wie und in welchem Umfang die Versicherten ihren Präferenzen für Krankenversicherungsschutz, was den Umfang oder auch konkrete Leistungsbündel betrifft, durch die Wahl ihrer Krankenkasse Ausdruck verleihen können. Es ist deshalb zu fragen, ob es möglich ist, zu effizienteren Aufteilungen der Versicherten auf die Krankenkassen zu gelangen und die Selbstselektion der Versicherten in der Sozialversicherung damit letztlich produktiv einzusetzen. Hierzu müsste nachgewiesen werden, dass durch die Selbstselektion der Versicherten innerhalb eines Sozialversicherungszweiges eine echte Pareto-Verbesserung zu erreichen ist, einzelne Individuen also bessergestellt werden können, ohne den Erwartungsnutzen anderer Sozialversicherter zu senken. Die vorliegende Analyse wird zeigen, dass dies auf der Basis eines halbwegs gut funktionierenden Risikoausgleichs möglich ist.

Zum Nachweis für die These, dass effizienzsteigernde Selbstselektionen von Versicherten unter bestimmten Voraussetzungen auch in der sozialen Krankenversicherung möglich sind, wird die Selbstaufteilung der sozial Krankenversicherten in zwei möglichen Dimensionen untersucht, nämlich hinsichtlich ihres in die Sozialversicherung eingebrachten Risikos und der Höhe ihres (versicherungspflichtigen) Einkommens. Hierbei ist die Einkommensdimension nur für die gesetzliche Krankenversicherung in Deutschland relevant, wo jede Krankenkasse ihre Beiträge einkommensabhängig gemäss ihres jeweiligen Beitragssatzes erhebt. Für die Schweizer Krankenversicherung bleibt die Möglichkeit der Selbstselektion nach guten und schlechten Risiken. Es ist im einzelnen darzustellen, wie die jeweiligen Selektionsmechanismen arbeiten und auf welche Weise sie in Verbindung mit einem funktionierenden Risikoausgleich zu einer Pareto-Verbesserung führen könnten. Es wird mit der Selbstselektion nach Risiko begonnen, weil sie in den existierenden Krankenversicherungssystemen beider Länder möglich wäre.

1) Vgl. z.B. Henke $(1996,448)$ und Breuer (1998, Kap. II). 


\section{Die Selbstselektion nach Risiko}

Um die möglichen nützlichen Auswirkung der Selbstselektion der Sozialversicherten nach Risiko zu analysieren, ist zunächst die Wirkung eines funktionierenden Risikoausgleichs herauszustellen. Die so beschriebene Situation bildet den Ausgangspunkt der weiteren Argumentation des Abschnitts 2.

\subsection{Die Auswirkungen eines funktionierenden Risikoausgleichs im theoretischen Modell}

Sofern der Risikoausgleich zwischen den Krankenkassen perfekt funktioniert, bewirkt er, dass jede Krankenkasse ihre Beiträge nicht danach bemessen muss, welche Risiken gerade bei ihr vorherrschend sind, sondern so kalkulieren kann, als wäre ein perfekter Durchschnitt aller Risiken bei ihr versammelt.

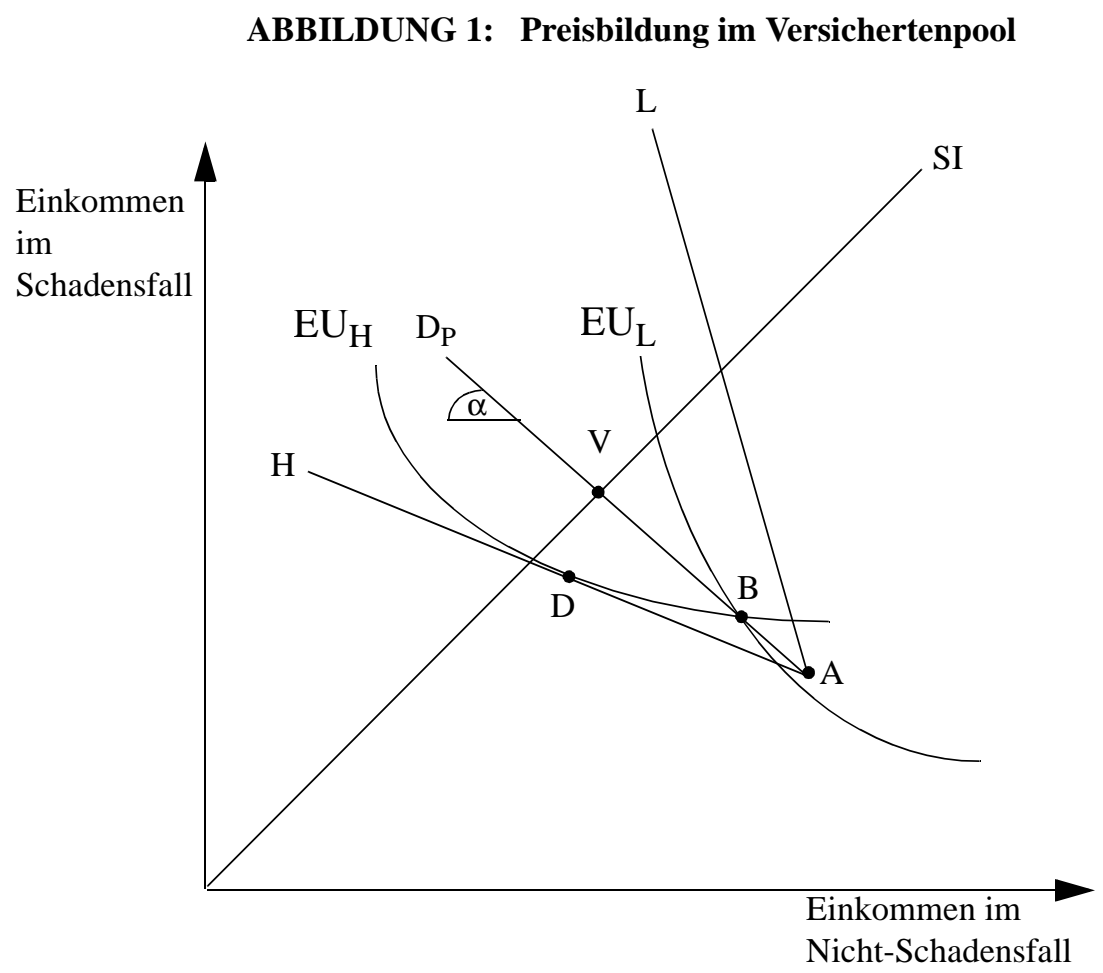

In Abbildung 1 kann die Wirkung des Risikoausgleichs wie folgt veranschaulicht werden: Eine Krankenkasse, die nur gute Risiken versichert, kann eine Einheit Versicherungsschutz (Einkommen wird aus dem Zustand des Nicht-Schadensfalls in den Zustand des Schadensfalls transferiert) zu einer Austauschrate anbieten, wie sie durch die Steigung der Geraden AL beschrieben wird. In dieser Austauschrate spiegele sich nicht nur das Versicherungsrisiko eines guten Risikos wider, sondern sie möge auch einen (proportionalen) Loading-Faktor beinhalten, welcher zur Deckung der administra- 
tiven Kosten der Versicherung notwendig ist. ${ }^{1}$ Eine Kasse, die ausschliesslich hohe Risiken zu versichern hat, kann dann (bei gleichem Loading-Faktor) den Versicherungsschutz nur zu einem ungünstigeren Austauschsatz anbieten, wie es durch die Steigung der Geraden AH graphisch repräsentiert wird. Für alle ,Mischungsverhältnisse ‘ zwischen guten und schlechten Risiken in einem Versichertenpool würden von A ausgehende Geraden relevant, die zwischen denen der Geraden AH und $\mathrm{AL}$ liegen. Die Gerade $\mathrm{AD}_{\mathrm{P}}$ möge das ,Mischungsverhältnis‘ widerspiegeln, das in der Grundgesamtheit der zu Versichernden herrscht. Funktioniert der Risikoausgleich perfekt ${ }^{2}$, wird jegliche bei einer einzelnen Kasse vorzufindende Abweichung von der Risikostruktur der Grundgesamtheit ausgeglichen, und es kann der einzelnen Kasse gleichgültig sein, welche Risikostruktur bei ihr gerade vorherrscht. Solange sicher gestellt ist, dass alle Versicherten den gleichen Deckungsgrad in Anspruch nehmen und Beiträge in der entsprechenden Höhe entrichten ${ }^{3}$, können die Kassen die Einheit Versicherungsschutz zu dem Austauschverhältnis anbieten, das durch die Gerade $\mathrm{AD}_{\mathrm{P}}$ repräsentiert wird. Der eingezeichnete Punkt B stellt nur eine mögliche Situation dar. Er repräsentiert eine Poolbildung, in der (vergleichbar mit der Situation in der Schweizer Krankenversicherung) alle Versicherten eine Teildeckung (der Deckungsgrad wird durch die Länge der Strecke AB veranschaulicht) zum festgelegten Preis erwerben müssen. ${ }^{4}$ Der Umfang der Subvention der schlechten Risiken durch die guten Risiken lässt sich entlang der Geraden $\mathrm{AD}_{\mathrm{P}}$ durch die staatliche Kontrollinstanz festlegen, die darüber entscheidet, welchen Deckungsgrad die Krankenkassen in der Grundversicherung anzubieten und die Versicherten zu erwerben haben. Alle gesetzlich vorgeschriebenen Ausgaben der Kassen, denen keine risikoäquivalenten Beiträge gegenüberstehen, müssen in den Risikoausgleich zwischen den Kassen eingehen.

Der Punkt B wird als Ausgangspunkt für die weiteren Überlegungen gewählt. In Punkt B erreichen die schlechten und die guten Risiken die Erwartungsnutzenniveaus $E_{\mathrm{H}}$ bzw. $\mathrm{EU}_{\mathrm{L}}$. In dieser Situation wäre eine Pareto-Verbesserung möglich, wenn gezeigt werden könnte, dass die guten Risi-

1) Um weiteren Komplikationen aus dem Weg zu gehen, wird der Loading-Faktor nicht nur als proportional angenommen, sondern es wird zusätzlich davon ausgegangen, dass er für gute wie für schlechte Risiken als gleicher Anteil vom Prämienvolumen erhoben wird.

2) Ein perfekt arbeitender Risikoausgleich setzt gerade voraus, dass die Informationsasymmetrien, die den Ausgangspunkt von Rotschild/Stiglitz (1976) bilden, überwunden sind. Die im folgenden zu analysierenden Separierungsgleichgewichte können folglich nicht auf unvollkommene Information zurückgeführt werden.

3) Der Deckungsgrad des Versicherungsschutzes kann ohne besondere Ausgleichsmassnahmen, wie sie unten diskutiert werden, nicht einfach der individuellen Wahl der Versicherten überlassen werden. Könnten die guten Risiken ihren Versicherungsschutz verringern, entfiele zumindest ein Teil der Einnahmeüberschüsse, die bei der Versicherung der guten Risiken anfallen. Diese ständen dann nicht mehr für die Subventionierung der schlechten Risiken zur Verfügung.

4) Wenn wir zunächst einmal von der Annahme ausgehen, dass die Individuen ausschliesslich Sozialversicherungsschutz nachfragen, stellt der Punkt B nur insofern eine Besonderheit dar, als er die unterste Deckungsrate eines Versicherungspools angibt, die ihren ,sozialen Zweck', nämlich die Besserstellung der hohen Risiken, erfüllt, erreichen doch in Punkt B die hohen Risiken den gleichen Erwartungsnutzen, wie sie ihn auch ohne Subventionierung in einem freien Versicherungsmarkt erreichen würden. Aufgrund des angenommenen Loading-Faktors würden die schlechten Risiken auch auf einem privaten Versicherungsmarkt keine Vollversicherung wählen. 
ken einen höheren Erwartungsnutzen erreichen könnten, ohne dass sie gleichzeitig ihre Subventionen an die schlechten Risiken senken müssten. Eine Reduktion des Preises pro Einheit Versicherungsschutz, den die niedrigen Risiken für den einheitlichen Versicherungsschutz zu zahlen haben, kommt daher für eine Pareto-Verbesserung nicht in Betracht, weil sonst auch die Subventionierung der schlechten Risiken reduziert würde. Eine Pareto-Verbesserung kann vielmehr nur dadurch erfolgen, dass die guten Risiken einen niedrigeren Deckungsgrad zu einem höheren Preis pro Einheit Versicherungsschutz wählen.

\subsection{Der Preis von Sozialversicherungsschutz für gute Risiken bei unterschiedlichen Deckungsgraden}

Die Zuschüsse für die schlechten Risiken, die einen Anteil von (1-a) am gesamten Versichertenbestand ausmachen mögen, werden im Ausgangspunkt des gemeinsamen Versichertenpools durch die Überschüsse im Versicherungsgeschäft mit den guten Risiken, deren Anteil a betrage, finanziert:

$a\left(P_{L}-\mu q_{L} s_{L} S\right)=(1-a)\left(\mu q_{H} s_{H} S-P_{H}\right)$

Das Symbol P steht für die Prämienzahlung. Die Symbole $\mu, q, s$ und S stehen für den LoadingFaktor, den Deckungsgrad, die Schadenseintrittswahrscheinlichkeit und die Schadenshöhe. Die Indizes L und H sollen kennzeichnen, dass die entsprechenden Grössen für die guten (low) und die schlechten (high) Risiken gemeint sind, wobei im folgenden von einer gleichen potentiellen Schadenshöhe für beide Risiken ausgegangen wird. Die Prämie, die ein gutes Risiko zu zahlen hat, um die Subventionierung der schlechten Risiken zu gewährleisten, berechnet sich demnach als:

$P_{L}=\frac{1-a}{a}\left(\mu q_{H} s_{H} S-P_{H}\right)+\mu q_{L} s_{L} S=\frac{1-a}{a} T+\mu q_{L} s_{L} S$

mit $T=\mu q_{H} s_{H} S-P_{H}$. In Gleichung (2) wird die von den guten Risiken zu zahlende Prämie zerlegt in einen Anteil, welcher der Deckung des eigenen Versicherungsrisikos (zuzüglich des Loading-Faktors) dient $\left(\mu q_{L} s_{L} S\right)$, und einen von jedem guten Risiko zu tragenden Anteil ( $\left.1 / a\right)$ am Transfervolumen, das zur Deckung des in der Sozialversicherung bei der Absicherung der schlechten Risiken entstehenden Defizits notwendig ist $[(1-a) T]$.

Das Austauschverhältnis, zu dem innerhalb der Sozialversicherung Einkommen im Nicht-Schadensfall gegen Einkommen im Schadensfall getauscht wird, ist in Abbildung 1 durch die Steigung der Geraden $\mathrm{AD}_{\mathrm{P}}$, ausgedrückt durch ihren jeweiligen Steigungswinkel $\alpha$, dargestellt. Die Grösse $\alpha$ wird allgemein definiert als der Quotient aus der Nettozahlung, die ein Versicherter im Schadensfall erhält, und seiner stets zu leistenden Prämienzahlung: 
$\alpha=\frac{q S-P}{P}$

Für die guten Risiken ergibt sich $\alpha$ durch Einsetzten der entsprechenden Werte aus Gleichung (2) in Gleichung (3). Hierdurch wird des möglich, $\alpha$ als Funktion des Deckungsgrades des guten Risikos, unter Konstanz des Transfers an das schlechte Risiko auszudrücken:

$$
\alpha=\frac{q_{L} S-\mu q_{L} s_{L} S-\frac{1-a}{a} T}{\mu q_{L} s_{L} S+\frac{1-a}{a} T} .
$$

Die Ableitung von Gleichung (4) nach $\mathrm{q}_{\mathrm{L}}$ ergibt die Änderung von $\alpha$, die das gute Risiko bei Aufrechterhaltung seiner Transfers hinzunehmen hat, wenn es seinen Deckungsgrad in der sozialen Krankenversicherung verändern will:

$$
\frac{\partial \alpha}{\partial q_{L}}=\frac{(1-a) a S T}{\left(a \mu q_{L} s_{L} S+(1-a) T\right)^{2}}>0
$$

Aus Gleichung (5) wird ersichtlich, dass sich das Austauschverhältnis (Einkommen im Schadensfall zu Einkommen im Nicht-Schadensfall) in der Sozialversicherung für ein gutes Risiko bei sinkendem $\mathrm{q}_{\mathrm{L}}$ immer verschlechtern muss. Anders ausgedrückt: Das gute Risiko hat pro Einheit Krankenversicherungsschutz einen höheren Preis zu zahlen, wenn es seinen Deckungsgrad in der sozialen Krankenversicherung verringern will, ohne dass die Transfers an die schlechten Risiken davon berührt werden.

Um welchen Betrag sich der Sozialversicherungsschutz für die guten Risiken verteuern muss, zeigt die Abbildung 2. Sie gibt drei mögliche Verläufe der Funktion $\alpha$ für das gute Risiko in Abhängigkeit von $\mathrm{q}_{\mathrm{L}}$ (Gleichung (4)) wieder bei folgenden angenommenen Werten für die anderen Grössen: $\mathrm{P}_{\mathrm{H}}=7, \mathrm{~s}_{\mathrm{H}}=0.5, \mathrm{~s}_{\mathrm{L}}=0.25$ und $\mathrm{S}=40$. Für $\mathrm{q}_{\mathrm{h}}$ wurden die Werte 0.4, 0.5 und 0.6 angenommen, um die Auswirkungen unterschiedlicher Transferströme innerhalb der sozialen Krankenversicherung auf die notwendige Veränderung von $\alpha$ zu zeigen.

Die Verringerung des Deckungsgrades entspricht einer Bewegung von rechts nach links auf den gezeigten Kurven. In der vorliegenden Situation ist die Mitgliedschaft der guten Risiken in der sozia-

1) Der Wert für $\alpha$ sollte realistischerweise nicht unter eins fallen, weil der Versicherte sonst eine Einheit seines Einkommens mit der Wahrscheinlichkeit von eins zu zahlen hätte, um im Schadensfall (dessen Wahrscheinlichkeit kleiner als eins ist) weniger als eine Einkommenseinheit ausgezahlt zu bekommen. Er könnte sich dann durch einen simplen Sparvorgang besserstellen. In den Abbildungen sind die Geraden zum Teil flacher gezeichnet, um die Übersichtlichkeit zu gewährleisten. 
ABBILDUNG 2: $\alpha$ bei verschiedenen Deckungsgraden $\left(q_{L}\right)$

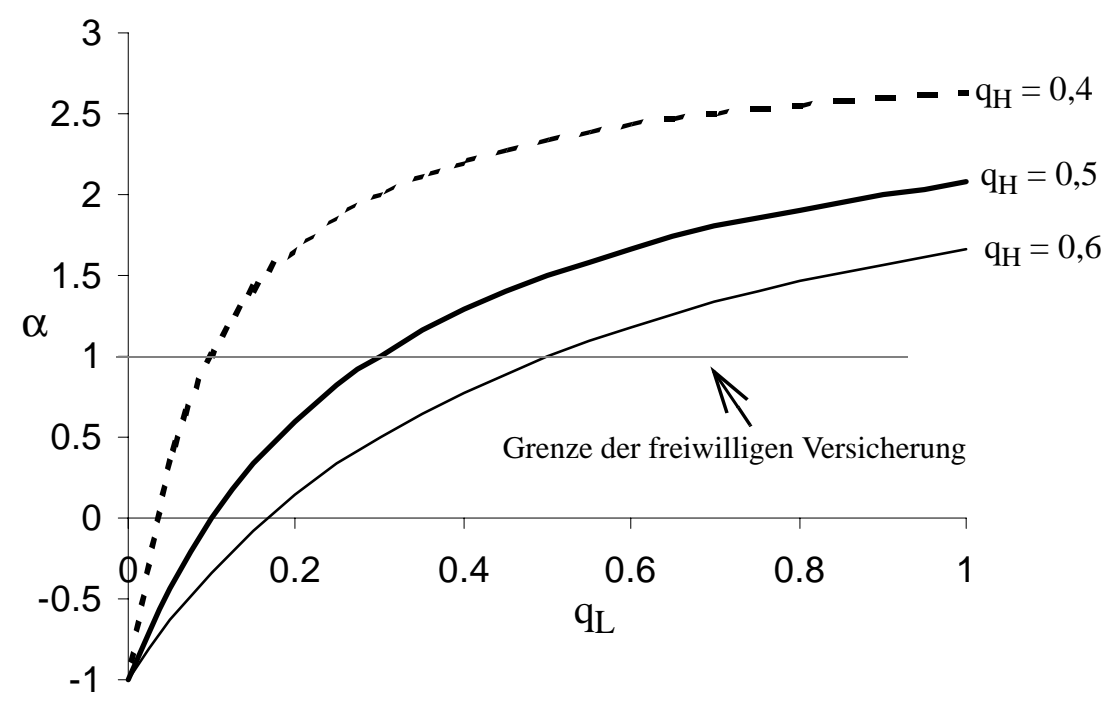

len Krankenversicherung nicht freiwillig, so dass es durchaus denkbar ist, dass $\alpha$ auch Werte von kleiner als eins annimmt, damit der Transfer an die schlechten Risiken gesichert ist. Theoretisch kann $\alpha$ bei den angenommenen Parameterwerten sogar negativ werden, was so zu interpretieren wäre, dass das gute Risiko im Schadensfall noch zusätzlich in die Versicherung einzuzahlen hätte, statt Leistungen von ihr zu bekommen, was dem Zweck einer Versicherung sicher zuwider läuft. Die für die Praxis relevantere Interpretation der Funktion lautet daher, dass es ab einem Deckungsgrad von Null für die guten Risiken nur noch möglich ist, die Transferzahlungen an die schlechten Risiken in der gleichen Höhe dadurch aufrecht zu erhalten, dass sie den ,Solidaranteil ' in die soziale Krankenversicherung in Form einer ,Sozialversicherungssteuer' einzahlen ohne Leistungen in Anspruch zu nehmen. Dass eine Reduzierung ihres in der Sozialversicherung in Anspruch genommenen Deckungsgrades für die guten Risiken durchaus rational sein kann, sie im Extremfall sogar völlig auf Leistungen verzichten werden, ist nun zu zeigen.

\subsection{Pareto-Verbesserung ohne Zusatzversicherung}

Im folgenden dieses Abschnitts und des Abschnitts 3 wird von der vereinfachenden Annahme ausgegangen, dass der Loading-Faktor in der Sozialversicherung nicht geringer ist als in der privaten Versicherung. Wie aus der Ableitung von Gleichung (2) nach $\mathrm{q}_{\mathrm{L}}$ hervorgeht, entsprechen in diesem Fall die Grenzkosten, die das gute Risiko zu tragen hat, um sein Absicherungsniveau zu erhöhen, dem Betrag, den es hierzu auch auf dem privaten Markt aufzubringen hätte. Sie bleiben von seiner Mitgliedschaft in der Sozialversicherung unberührt: 
$\frac{\partial P_{L}}{\partial q_{L}}=\mu s_{L} S$

Die Konsequenzen der Gleichung (6) sind in Abbildung 3 wiedergegeben. Abbildung 3 stellt einige Details aus der Abbildung 1 nochmals dar, wobei der besseren Übersicht wegen die Ordinate gestreckt wurde. Aus der Abbildung 1 tauchen die Punkte A und B, sowie die Geraden AL und $\mathrm{AD}_{\mathrm{P}}$

\section{ABBILDUNG 3: Die Auswirkung von Wahlmöglichkeiten auf die Höhe des Sozialversicherungsschutzes}

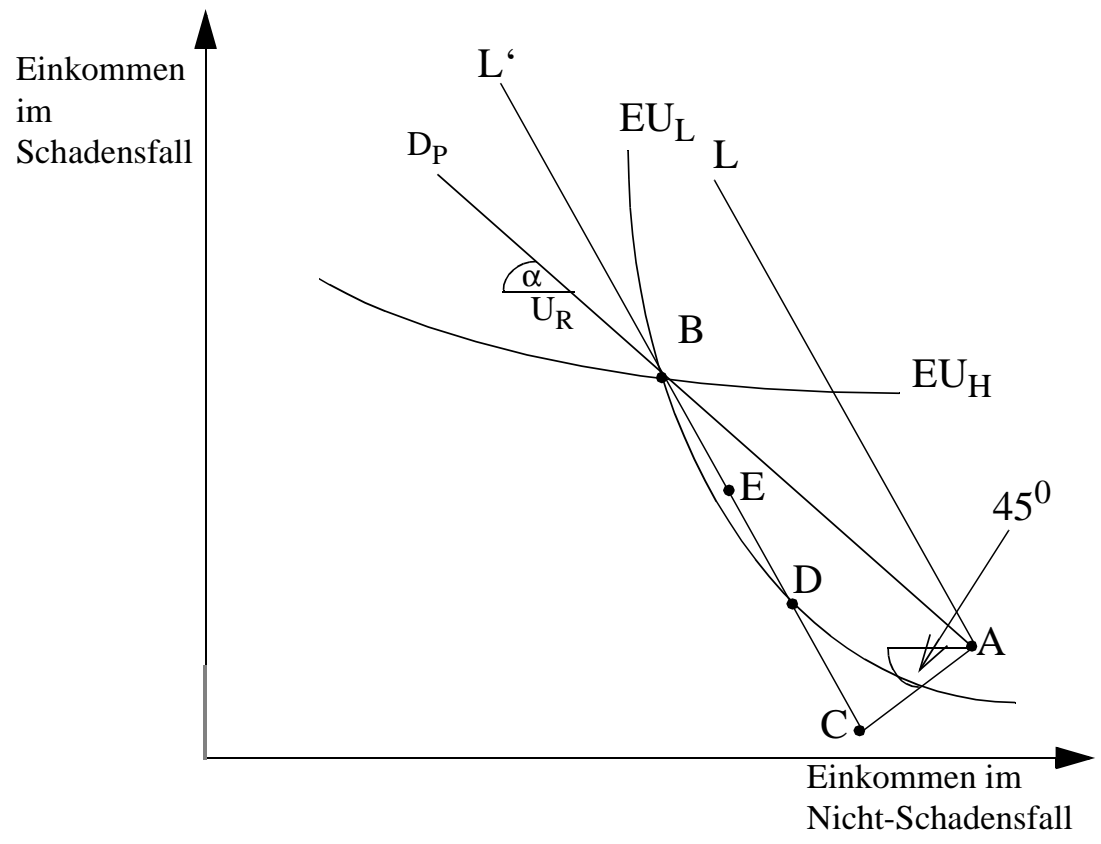

wieder auf. Der zusätzlich eingezeichnete Punkt $\mathrm{C}$ stellt die Einkommensminderung eines guten Risikos dar, wie sie sich durch den Zwangstransfer an die schlechten Risiken ergibt. Dadurch dass der Transfer unabhängig von der eigenen Wahl des Deckungsgrades erfolgt, wirkt er wie eine Steuer, die das Einkommen des transferpflichtigen Individuums im Schadens-, wie im Nichtschadensfall gleichermassen reduziert. Die zu AL parallel verlaufende Gerade CL' repräsentiert die Kombinationen von Einkommen im Nicht-Schadensfall zu Einkommen im Schadensfall, die ein gutes Risiko in der Sozialversicherung wählen kann, ohne dass es seinen Transfer an die schlechten Risiken reduziert. Formal ist diese Beziehung durch die Gleichung (2) erfasst. Sie sagt aus, dass die guten Risiken bei gleichen Loading-Faktor in der Privat- und Sozialversicherung ihren Versicherungsschutz zu den gleichen Grenzkosten oder Grenzersparnissen verändern können, wie ausserhalb der Sozialversicherung. In der Praxis würde das so aussehen, dass verschiedene Krankenkassen unterschiedliche Leistungspakete zu unterschiedlichen Prämien anbieten. Die konkurrenzfähigen Angebote aller Kassen zusammen würden in Abbildung 3 entlang der Geraden CL' zu finden sein. 
Aus der Abbildung 3 wird deutlich, dass es für das Individuum möglich ist, entlang der Geraden CL' eine höhere Indifferenzkurve als die ursprüngliche zu erreichen, indem es einen Tangentialpunkt mit einer seiner Indifferenzkuren im Bereich zwischen den Punkten B und D anstrebt, z.B. Punkt E. Die guten Risiken können sich somit besserstellen, indem sie den ursprünglich gemeinsamen Versicherungspunkt B verlassen und auf einen Teil des Sozialversicherungsschutzes verzichten. Der Verzicht kann je nach Gewicht des Loading-Faktors und der Risikofreude des Individuums sogar vollständig sein. In diesem Falle würde das Individuum den Transfer T in Form einer ,Krankenversicherungssteuer' einzahlen, ohne praktisch einen nennenswerten Versicherungsschutz in der sozialen Krankenversicherung bereitgestellt zu bekommen. ${ }^{1}$

Der Wettbewerb um die effizienten Kombinationen zwischen Prämienzahlung und Deckungsgrad für die guten Risiken wird dafür sorgen dass in der Sozialversicherung keine Tarife mit einem Deckungsgrad höher als B angeboten werden (sie würden von den schlechten Risiken gewählt, aber die dann fälligen Ausgaben der Krankenkassen nicht in voller Höhe im Risikoausgleich berücksichtigt) und die Reduktion der gesamten Prämienzahlung der guten Risiken nicht höher ausfällt als die durch die Gerade CL' erlaubte, damit sie sich dem Solidarausgleich nicht entziehen können. Die Versicherten würden dann, wie in Abbildung 3 dargestellt, das Angebot wählen, welches ihren Präferenzen am nächsten kommt. Durch ihre jeweiligen Angebote können die Krankenkassen somit auch bestimmte Risiken an sich binden, was den Anreizen für die Versicherten entgegenwirkt, bei nicht perfekt funktionierendem Risikoausgleich zu den Krankenkassen mit jeweils günstiger Risikostruktur zu wechseln. Insofern würde die Selbstselektion der Versicherten auch zur Stabilität des gesamten Versicherungssystems beitragen.

Um in jedem Fall sicher zu stellen, dass die Transferzahlungen an die schlechten Risiken bei beliebiger Senkung des Deckungsgrades durch die guten Risiken auch tatsächlich in voller Höhe beibehalten werden, und durch die Wahlentscheidung der guten Risiken nicht einfach nur eine Umverteilung erfolgt, bedarf es einer besonderen Konstruktion des Risikoausgleichs. Bevor die guten Risiken den von ihnen in Anspruch genommenen Deckungsgrad verringern, ist der Risikoausgleich in Höhe des extern festgelegten Grundsicherungsniveaus vorzunehmen, das den schlechten Risiken in der Sozialversicherung geboten werden soll, um ihre Situation zu verbessern. Für die Ermittlung der Nettotransfers zwischen den Kassen ist hierbei zunächst davon auszugehen, dass alle Versicherten, gleich welchen Risikos, den Ausgangsdeckungsgrad B in Anspruch nehmen. Erst wenn die Nettotransfers zwischen den Krankenkassen feststehen, dürfen die Kassen verschiedene Tarife mit verschiedenen Deckungsgraden anbieten und damit versuchen, Versicherte an sich zu binden. Es ist sogar durchaus möglich, dass verschieden effizient arbeitende Kassen auch unterschiedliche Min-

1) Die in der Schweizer Krankenversicherung bestehende Möglichkeit, unterschiedliche Franchise-Sätze zu wählen geht in die in diesem Beitrag skizzierte Richtung der Risikoselektion, allerdings ohne die Möglichkeit des völligen Verzichts auf Sozialversicherungsleistungen einzuräumen. Die Analyse zeigt, dass man diesen Weg der Risikoselektion weiter gehen kann und, bei Wahl der paretianischen Wohlfahrtstheorie als normativer Masstab, auch sollte. 
destbeiträge für Individuen vorsehen, die keine (oder fast keine) Versicherungsleistungen in Anspruch nehmen. Damit würde auch ein Wettbewerb der Krankenkassen um die geringste ,Krankenversicherungssteuer` entstehen, der aber unter Verteilungsgesichtspunkten unbedenklich ist.

Zusammenfassend kann somit gesagt werden, dass eine durch einen Risikoausgleich flankierte Selbstselektion der Krankenversicherungsmitglieder nach Risiko zu eindeutigen Pareto-Verbesserungen führen kann, ohne den angestrebten sozialen Ausgleich in Frage zu stellen. Die durch das Wandern der guten Risiken entstehende Selbstselektion von Versicherten erscheint damit in einem positiven Licht, und die in der Literatur über das cream-scimming herausgestellten Nachteile einer Risikoselektion in der Sozialversicherung können relativiert werden. Die Selbstselektion kann sich sogar eindeutig wohlfahrtssteigernd auswirken, dieses auch im Rahmen der Sozialversicherung, die letztlich auf der Idee eines einheitlichen (solidarischen) Risikopools beruht.

\subsection{Pareto-Verbesserung mit Zusatzversicherung}

Während in den oben beschriebenen Fällen der von der Sozialversicherung verbindlich vorgeschriebene Versicherungsschutz immer noch einen so hohen Deckungsgrad umfasste, dass ein gutes Risiko zum durch die Gerade CL' beschriebenen Austauschverhältnis freiwillig nur ein niedrigeres Absicherungsniveau wählen würde, sind auch andere Situationen denkbar. So kann der in der Sozialversicherung gebotene Versicherungsschutz derart gering ausfallen, dass auch die guten Risiken zu den Grenzkosten, die sie auf dem Markt vorfinden, eine Ausweitung ihres Versicherungsschutzes anstreben. Ein solcher Fall ist in Abbildung 4 skizziert. In ihr werden die aus Abbildung 3 vertrauten Punkte A, B und $\mathrm{C}$ übernommen sowie die Geraden $\mathrm{AC}, \mathrm{AD}_{\mathrm{P}}$ und $\mathrm{CL}$ ' noch einmal abgetragen. $\mathrm{Im}$ Vergleich zur Abbildung 3 sind die Präferenzen des guten Risikos nun aber so, dass es zum in der Sozialversicherung herrschenden Preisverhältnis $\alpha$ zwar einen geringeren Versicherungsschutz nachfragen würde, entlang der nach Gleichung (2) relevanten Budgetgerade aber durchaus gewillt wäre, seinen Versicherungsschutz sogar noch auszudehnen. Die optimale Reaktion dieses Individuums wäre es, den gebotenen Sozialversicherungsschutz am Punkt B voll in Anspruch zu nehmen und sich privat weiter abzusichern, bis sein Erwartungsnutzenoptimum (ein Punkt auf der Gerade CL' oberhalb von B) erreicht ist. Im theoretischen Grenzfall einer privaten Versicherungsmöglichkeit, die aktuarisch faire Prämien ohne einen Loading-Faktor verlangt, würde ein gutes Risiko stets diese Strategie wählen und seinen Versicherungsschutz auf dem privaten Markt bis zur vollständigen Dekkung seines Risikos, also bis zur in Abbildung 1 wiedergegebenen Vollversicherungsgerade aufstokken.

Ob eine private Zusatzversicherung in Anspruch genommen wird, hängt damit ausschliesslich von der Höhe des insgesamt von dem guten Risiko gewünschten Versicherungsschutzes bei einem durch die Gerade CL' wiedergegebenen Preisverhältnis ab. Will sich das Individuum nur bis zum Punkt B versichern, wird es ausschliesslich Sozialversicherungsschutz in Anspruch nehmen, weil 


\section{ABBILDUNG 4: Die Auswirkung von Wahlmöglichkeiten unter der Option einer Zusatzversicherung}

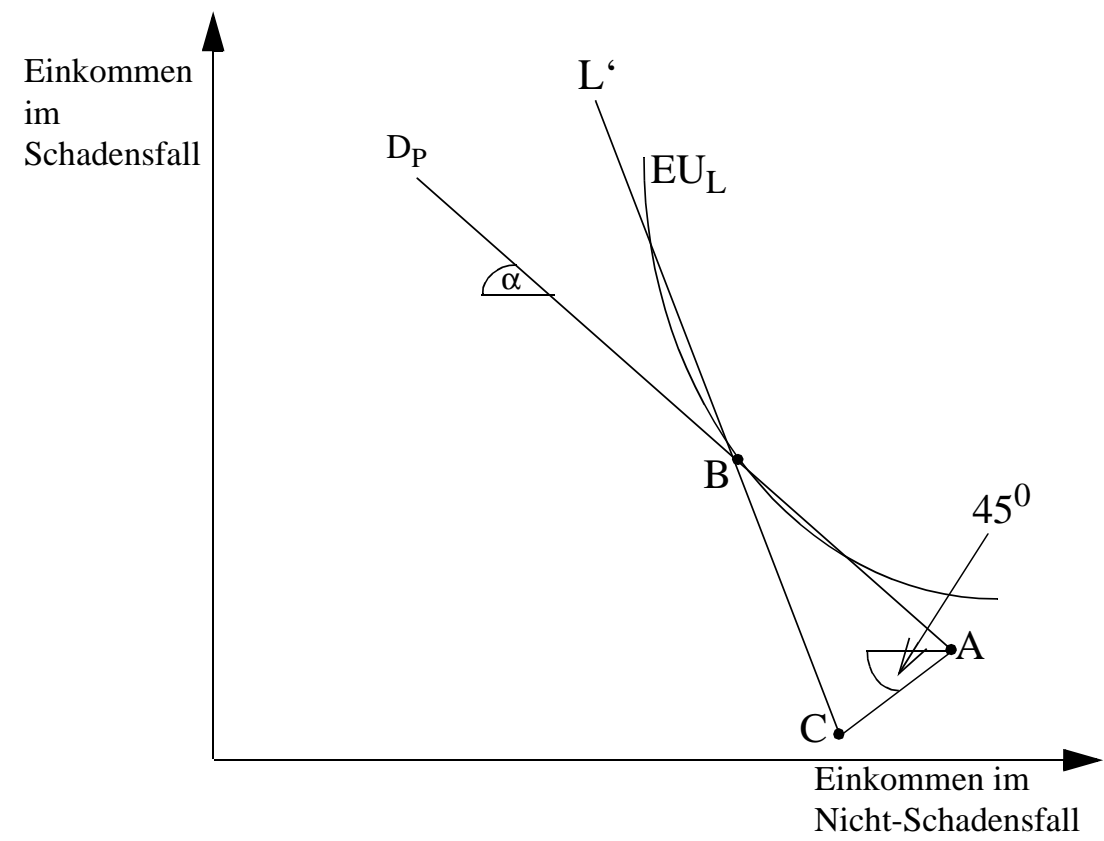

dieser pro Einheit Versicherungsschutz mit zunehmendem Deckungsgrad immer günstiger wird (Gleichung (5)). Erst bei einem gewünschten Deckungsgrad über den Punkt B hinaus wird es erforderlich, weiteren Versicherungsschutz im Rahmen einer Zusatzversicherung nachzufragen. Ob dies bei dem gleichen Anbieter geschieht, der auch die Grundversicherung zur Verfügung stellt, kann dem Individuum gleichgültig sein. Entscheidend ist, dass der Anbieter der Zusatzversicherung keinen höheren Preis pro Einheit Versicherungsschutz verlangt, als den durch die Gerade CL' repräsentierten.

Es sollte betont werden, dass die Festlegung des von der Sozialversicherung gebotenen und von den schlechten Risiken auf jeden Fall in Anspruch genommenen Deckungsgrades in dem vorgestellten Modell ausschliesslich aus Verteilungserwägungen erfolgt. Unabhängig von der hier diskutierten gewollten Verbilligung des Versicherungsschutzes für schlechte Risiken kann es für den Gesetzgeber aber auch sinnvoll sein, ein minimales Versicherungsniveau für alle Individuen vorzuschreiben, gleich welches Versicherungsrisiko sie bergen. Einzelne Individuen könnten sonst versucht sein, auf ihre eigene Vorsorge durch den Abschluss eines Versicherungsvertrages zu verzichten, um dann im Schadensfall die Leistungen der Sozialhilfe in Anspruch zu nehmen (Buchholz/Wiegard 1992) ${ }^{1}$. Für die guten Risiken heisst das, dass aus allokativen Erwägungen auch sie nicht den Versicherungs-

1) Die von Buchholz/Wiegard (1992) angesprochene Frage stellt kein reines Verteilungsproblem dar. Weil diejenigen Individuen, die auf einen Versicherungsschutz verzichten würden, mit ihrer Entscheidung im gesamten Sicherungssystem Externalitäten verursachen würden, käme es zu Ineffizienzen, die auch rein allokativ zu bewerten sind. 
schutz auf Null senken können und zumindest eine minimale Absicherung (z.B. für längere Krankenhausaufenthalte oder grössere Operationen) nachweisen können sollten. Aufgrund der obigen Analyse spricht nichts dagegen, auch die guten Risiken zu verpflichten, die aus dem Buchholz-Wiegard-Argument folgende minimale Absicherung in der Sozialversicherung zu suchen. Durch ein Opting-Out aus der Sozialversicherung könnten sie sich nicht besserstellen.

\section{Selbstselektion der Versicherten nach Einkommen}

Die nun zu diskutierende Selbstselektion der Versicherten nach Einkommen kann zu Pareto-Verbesserungen in Krankenversicherungssystemen mit einkommensabhängigen Beiträgen, wie z.B. in der Gesetzlichen Krankenversicherung (GKV) der Bundesrepublik Deutschland führen, wieder ohne den Solidarausgleich in Frage zu stellen. Die vorzustellende Argumentation weist manche Analogie zur oben diskutierten Selbstselektion nach Risiko auf, aber auch einen deutlichen Unterschied, der eine gesonderte Behandlung dieser Variante der Selbstselektion rechtfertigt.

\subsection{Unterschiedliche Versicherungspreise in der GKV}

Der wesentliche Unterschied zur vorangegangenen Analyse ist der, dass die GKV-Versicherten entsprechend ihren unterschiedlichen versicherungspflichtigen Einkommen auch unterschiedliche Preise für ihren Sozialversicherungsschutz zahlen, wie in Abbildung 5 verdeutlicht.

In Abbildung 5 sind die Versicherungsmöglichkeiten für ein ,armes ‘ und ein ,reiches“ Individuum dargestellt. Um die Diskussion in diesem Abschnitt 3 auf die Einkommensunterschiede zwischen den Individuen zu konzentrieren, wird davon ausgegangen, dass beide Versicherten das gleiche Risiko aufweisen. Entsprechend dieser Annahmen ergibt sich folgendes Bild: Die Ausgangspunkte für das arme und das reiche Individuum $\left(A_{P}\right.$ und $\left.A_{R}\right)$ sind unterschiedlich. Das reiche Individuum verfügt sowohl im Schadens- als auch im Nicht-Schadensfall über ein höheres Einkommen als das arme Individuum. Weil beide Individuen gleich gute oder schlechte Risiken darstellen, haben die Geraden, die das Austauschverhältnis zwischen Einkommen im Schadensfall zu Einkommen im Nichtschadensfall beschreiben $\left(A_{P} P\right.$ und $\left.A_{R} R\right)$, die gleiche Steigung. Sie stellen allerdings nicht die in der sozialen Krankenversicherung relevanten Geraden dar. Dadurch dass die Beiträge bei gleichem Beitragssatz für alle Versicherten einer Krankenkasse einkommensabhängig erhoben werden, zahlt das reiche Individuum pro Einheit Versicherungsschutz mehr als das arme. Der Arme kann damit zu einem günstigeren Austauschverhältnis Einkommen vom Nicht-Schadensfall in den Schadensfall transferieren. Das reiche Individuum findet hingegen ein ungünstigeres Austauschverhältnis vor als dasjenige, das sich bei einer Beitragsbemessung ergeben würde, die sich (wie oben) am individuellen Risiko des Versicherten zuzüglich eines Loading-Faktors orientiert. Die Geraden $A_{P} D_{P}$ und $A_{R} D_{R}$ veranschaulichen diesen Sachverhalt. Wenn wir dieses Mal davon ausgehen, dass die 


\section{ABBILDUNG 5: Unterschiedliche Preise pro Einheit Versicherungsschutz} in der GKV

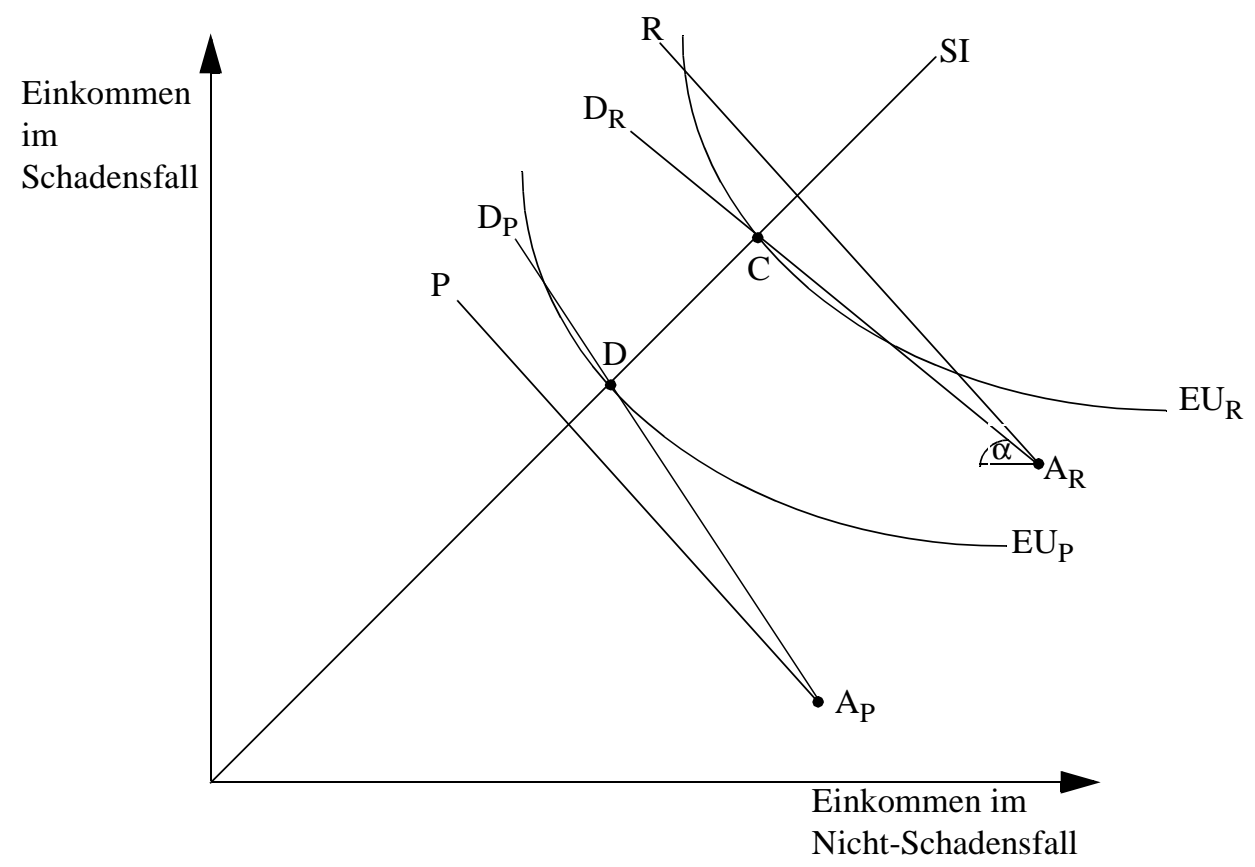

Nach: Strassl (1988, 232)

GKV in der Ausgangssituation eine Vollversicherung bietet, erreichen die beiden Individuen die Erwartungsnutzen, welche durch die Indifferenzkurven $\mathrm{EU}_{\mathrm{P}}$ und $\mathrm{EU}_{\mathrm{R}}$ repräsentiert sind. Es ist in der Abbildung auch zu erkennen, dass sich die niedrigen Einkommen bei dem in der GKV herrschenden Preis für ihren Krankenversicherungsschutz überversichern würden, wenn ihnen dies erlaubt wäre. Die hohen Einkommen würden hingegen zu dem in der GKV für sie massgeblichen Preis nur eine geringere Deckung als in der Privatversicherung wählen. Um die Subventionierung der niedrigen Einkommen durch die hohen Einkommen in der GKV unabhängig von der Aufteilung der Versicherten auf die einzelnen Krankenkassen sicher zu stellen, wird die Höhe der versicherungspflichtigen Einkommen ${ }^{1}$ im Risikoausgleich der deutschen GKV berücksichtigt.

\subsection{Der Preis des Sozialversicherungsschutzes für hohe Einkommen bei unterschiedlichen Deckungsgraden}

Analog zum Vorgehen in Abschnitt 2 soll ermittelt werden, welche Preisveränderung für eine Einheit Versicherungsschutz ein Reicher bei Konstanz seines Transfers an die Armen hinzunehmen hat, wenn er seinen Deckungsgrad senken möchte, um Beiträge zur GKV zu sparen. Da die Subventio-

1) Zur Beitragsbemessung wird nur das Einkommen der Versicherten aus unselbständiger Erwerbstätigkeit bis zu einer Beitragsbemessungsgrenze, die in der GKV zugleich die Versicherungspflichtgrenze darstellt, zugrundegelegt. Nach Überschreiten dieser Einkommensgrenze kann der Versicherte wählen, ob er weiterhin Mitglied der GKV bleiben möchte oder nicht. Im letzteren Fall entzieht er sich den Umverteilungsmechanismen der GKV völlig. 
nen für die Krankenkasse des Armen aus den Überschüssen der Krankenkasse des Reichen finanziert werden, gilt als Budgetrestriktion der Krankenkasse:

$a\left(b_{R} E_{R}-\mu q_{R} S\right)=(1-a)\left(\mu q_{A} s S-b_{A} E_{A}\right)=(1-a) T$,

wobei mit $\mathrm{E}_{\mathrm{R}}$ und $\mathrm{E}_{\mathrm{A}}$ die Einkommen des Armen und des Reichen gemeint sind. $\mathrm{q}_{\mathrm{R}}$ und $\mathrm{q}_{\mathrm{A}}$, die den Deckungsgrad der Krankenversicherung widerspiegeln sollen, sind für beide Individuen in der Ausgangssituation identisch, ebenso wie deren Beitragssätze $\left(b_{R}\right.$ und $\left.b_{A}\right)$ und deren Schadenseintrittswahrscheinlichkeit und Schadenshöhe (s und $\mathrm{S}$ ). Die Grössen s und S und $\mu$, das wieder für den Loading-Faktor steht, werden ohne Indizes geschrieben, weil sie annahmegemäss für beide Individuen gleich sind und bleiben. Mit a sei jetzt der Anteil der hohen Einkommen an der Gesamtzahl der GKV-Versicherten bezeichnet. In der Grösse T ist wieder die Transfersumme zusammengefasst, welche dieses Mal von den Reichen an die Bezieher niedriger Einkommen in der GKV fliesst. Wie man durch Auflösen der Gleichung (7) nach $b_{R} E_{R}$ leicht zeigen kann, setzt sich auch hier die Beitragszahlung des Reichen zusammen aus einem Betrag, den er für seinen eigenen Versicherungsschutz und seinen Anteil am Transfer an die Armen in der GKV aufwendet:

$$
b_{R} E_{R}=\frac{(1-a) T}{a}+\mu q_{R} S S
$$

Analog zu Abschnitt 2 ist es möglich, das Austauschverhältnis von Einkommen im Schadensfall zu Einkommen im Nichtschadensfall (und damit indirekt den Preis pro Einheit Versicherungsschutz) anzugeben. Es entspricht der Steigung der Geraden $A_{R} D_{R}$ und beträgt:

$$
\alpha=\frac{q_{R} S-b_{R} E_{R}}{b_{R} E_{R}}
$$

Dieser Versicherungspreis lässt sich unter der Bedingung, dass ein konstanter Transfer an den Armen fliesst, wieder als Funktion von $q_{R}$ ausdrücken. Aus Gleichung (9) in Verbindung mit Gleichung (7) folgt:

$$
\alpha=\frac{q_{R} S(1-\mu s)-\frac{1-a}{a} T}{\mu q_{R} s S+\frac{1-a}{a} T},
$$

und die Ableitung von Gleichung (10) nach $\mathrm{q}_{\mathrm{R}}$ ergibt: 
$\frac{\partial \alpha}{\partial q_{R}}=\frac{(1-a) a S T}{\left(a \mu q_{R} s S+(1-a) T\right)^{2}}>0$.

Die Gleichung (11) ist analog der Gleichung (5) so zu interpretieren, dass sich Bedingungen für den Transfer von Einkommen vom Nichtschadensfall in den Schadensfall für den Reichen mit abnehmendem Deckungsgrad immer verschlechtern. Das Resultat ist somit völlig analog der in Abschnitt 2 durchgeführten Analyse.

\subsection{Mögliche Pareto-Verbesserungen}

Ebenso kann auch analog begründet werden, warum der Reiche bei Senkung seines Deckungsgrades trotz der damit einhergehenden Preiserhöhung für die Einheit Sozialversicherungsschutz einen Zuwachs an Erwartungsnutzen realisieren kann. Abbildung 6 stellt die sich ergebende Situation dar.

\section{ABBILDUNG 6: Erwartungsnutzengewinne für den Reichen durch Wahl} des Deckungsgrades in der GKV

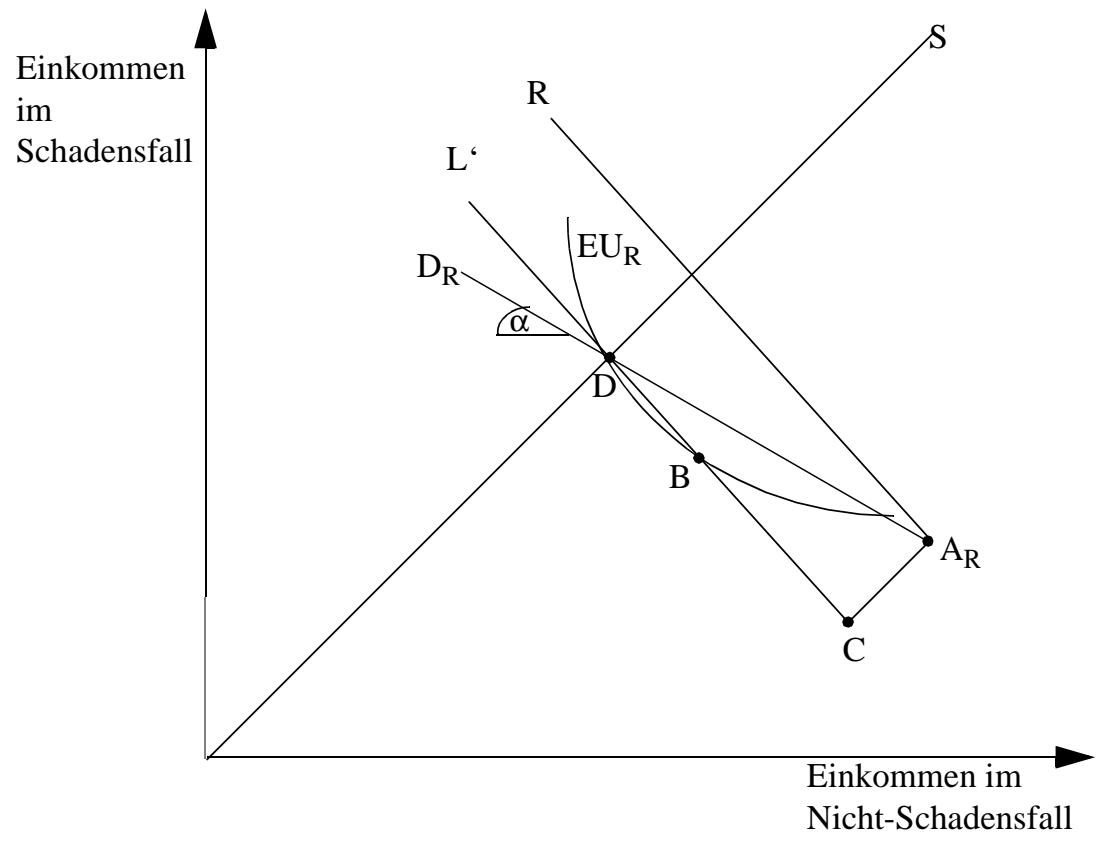

Der zwangsweise Transfer eines Reichen an die Armen bewirkt, dass er sich ohne Versicherung an Punkt C befinden würde. Gemäss Gleichung (8) wird die Gerade CL` zur relevanten Budgetrestriktion für ihn. Da wir den speziellen Fall betrachten, dass die GKV in der Ausgangssituation eine Vollversicherung bot, kann das Individuum auf jeden Fall durch die Senkung seines Deckungsgrades einen höheren Erwartungsnutzen realisieren, stellt doch die Vollversicherung bei nicht aktuarisch fairer Versicherungsprämie nie die optimale Wahl für ein Individuum mit einer von-Neuman-Morgenstern-Nutzenfunktion dar. In Abbildung 6 wird es vielmehr sein Optimum auf der Geraden CL 
zwischen den Punkten B und D finden. Je nach Höhe des Loading-Faktors ist es natürlich auch wieder denkbar, dass praktisch keine Leistungen der GKV in Anspruch genommen werden und das Individuum eine reine ,Krankenversicherungssteuer` zahlt. In jedem Fall aber kann sich das reiche Individuum verbessern, ohne seinen Transfer an den Armen (und damit letztlich dessen Erwartungsnutzen) zu reduzieren, was eine eindeutige Pareto-Verbesserung impliziert.

Wenn die GKV nur eine Teilsicherung anbietet, kann es auch für die Bezieher hoher Einkommen lohnend erscheinen, sich neben der vollen Inanspruchnahme des in der GKV gebotenen Versicherungsschutzes wieder zusätzlich zu versichern. Die Argumentation ist völlig analog der in Abschnitt 2.4 vorgestellten und muss daher hier nicht wiederholt werden.

\section{Das Zusammenspiel von Privat- und Sozialversicherung bei unterschiedlichen Loading-Faktoren}

Wurden die Resultate in den Abschnitte 2 und 3 unter der Annahme abgeleitet, dass sich die Loading-Faktoren in der Privat- und Sozialversicherung nicht voneinander unterscheiden, ist realitätsnäher davon auszugehen, dass die Kosten pro Einheit Versicherungsschutz in beiden Versicherungsarten durchaus unterschiedlich sein können. Insbesondere ist zu berücksichtigen, dass die Sozialversicherung ihre Mitglieder nicht aufwendig akquirieren muss und dadurch gegenüber der Privatversicherung, die ein umfangreiches Verkaufsnetz zu unterhalten hat, über erhebliche Kostenvorteile verfügt (Mitchell/Zeldes 1996, 366). Die graphischen Konsequenzen der sich hieraus ergebenden Situation sind in Abbildung 7 wiedergegeben. Sie stellt die Situation für ein reiches GKVMitglied dar, welches auf dem privaten Versicherungsmarkt ein durch die Gerade AR repräsentiertes Austauschverhältnis vorfinden würde. Die Sozialversicherung möge eine Teildeckung bis zum Punkt B bieten, so dass $\alpha$ das in der Ausgangssituation herrschende Austauschverhältnis für den Sozialversicherungsschutz wiedergibt. Durch die für die Sozialversicherung angenommenen Kostenvorteile jedoch kann das Individuum innerhalb eines Kontinuums an Beitrags-Leistungspaketen wählen, das auf der Linie CBL' zu finden ist. Solange es einen Deckungsgrad wählt, der unter dem von B liegt Abschnitt CB), liegen die Grenzkosten des Versicherungsschutzes niedriger als in der privaten Versicherung. Erst wenn der von der Sozialversicherung gebotene höchste Deckungsgrad überschritten wird (Abschnitt BL'), muss der Versicherte die höheren Grenzkosten in Kauf nehmen, die er in gleicher Höhe auch in einer privaten Versicherung vorfinden würde (der Abschnitt BL ' verläuft parallel zur Geraden AR). Wie sich anhand der Abbildung leicht erschliesst, muss ein Individuum unter diesen Kostenbedingungen eine starke Präferenz für zusätzlichen Versicherungsschutz haben, um sich von den gestiegenen Grenzkosten für weiteren Versicherungsschutz ab Punkt B nicht von dem Erwerb weiterer Einheiten abhalten zu lassen. Umgekehrt sinken die Versicherungsbeiträge bei Rückführung des Deckungsgrades von Punkt B nicht so stark, wie sie in der privaten Sicherung sinken würden. Der geknickte Verlauf der Kurve CBL' in Abbildung 7 kann somit ein Erklärungsele- 


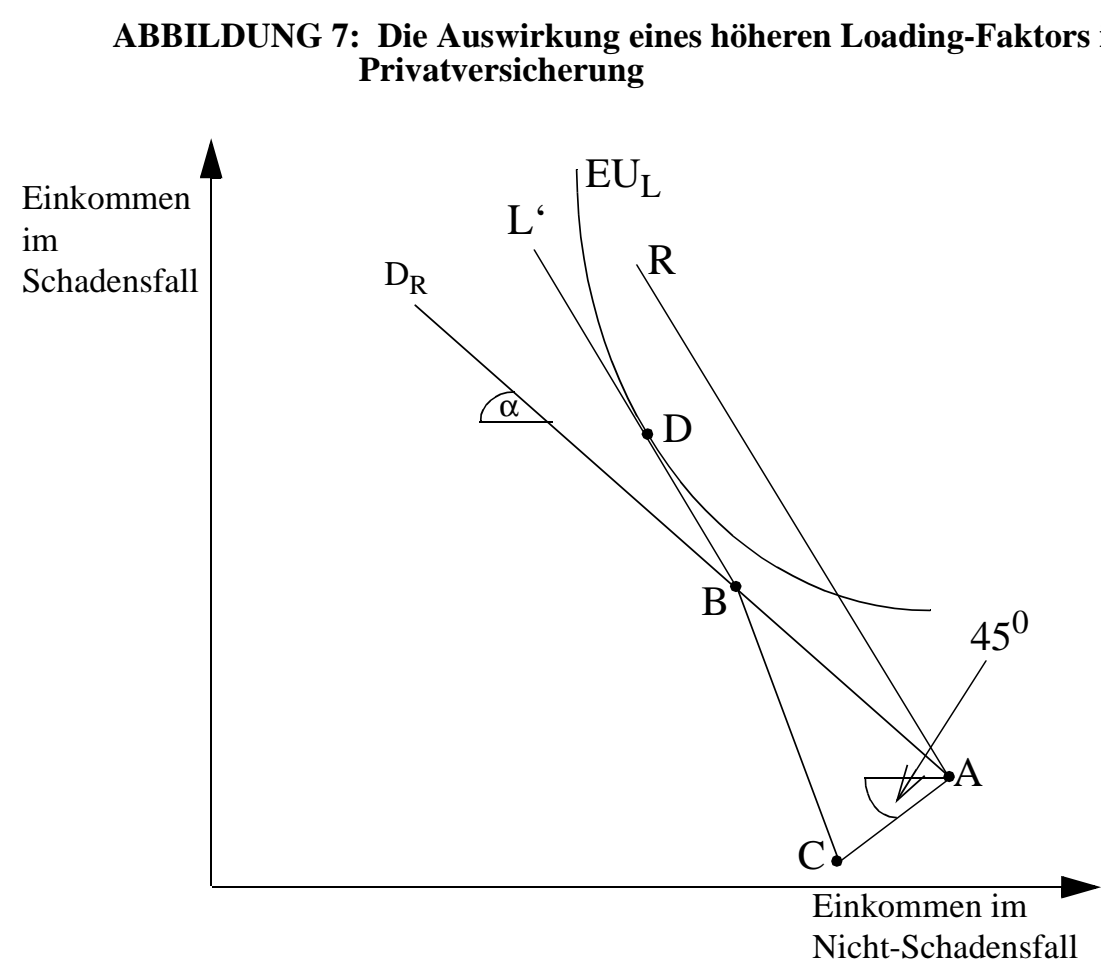

ment dafür bilden, warum in der Praxis der Übergang zwischen der sozialen Grundversorgung zur privaten Zusatzversicherung in der Krankenversicherung gerade nicht so fliessend ist, wie die Analyse in den Abschnitten 2 und 3 nahelegt. Ferner legt die geknickte Preisgerade nahe, dass auch unter erweiterten Wahlmöglichkeiten für die Versicherten in der Sozialversicherung damit zu rechnen ist, dass ein Grossteil der Versicherten das ursprüngliche Absicherungsniveau beibehalten wird.

Weitere Möglichkeiten der Kostendifferenzierung zwischen einzelnen Anbietertypen in der Sozial- und Privatversicherung können leicht in die Darstellung integriert werden. Z.B. ist es denkbar, dass eine Versicherung, die ausschliesslich eine Zusatzversorgung anbietet, einen Kostennachteil gegenüber den Versicherungen hat, die auf einen Kundenstamm in der Grundversorgung zurückgreifen können. Das hier vorgestellte Instrumentarium würde auch eine Analyse solche Fälle erlauben. Die Grundaussage aber, dass mit der Selbstselektion der Sozialversicherten Pareto-Verbesserungen zu erzielen sind, wird dadurch nicht in Frage gestellt.

\section{Zusammenfassung}

Das Ziel dieses Beitrages war es zu zeigen, dass einheitliche Pool-Lösungen, wie sie unter anderem zur Lösung von Problemen der adversen Selektion durchgeführt werden, nicht optimal sein müssen. Vielmehr kann eine durch einen Risikoausgleich abgestützte Selbstselektion von Versicherten nach Risiko und Einkommen auch in einer die individuellen Risiken zunächst nicht berücksichtigenden 
Sozialversicherung zu allokativen Verbesserungen führen. Ein vielleicht überraschendes Ergebnis der vorgestellten Analyse lautet, dass es für gute Risiken bzw. hohe Einkommen in der GKV rational sein kann, auf Teile des Sozialversicherungsschutzes zu verzichten, obwohl die Einheit Sozialversicherungsschutz für sie durch diese Wahl teurer wird. Derzeit sind z.B die guten Risiken zur Aufrechterhaltung der Transfers an die schlechten Risiken dazu gezwungen, einen bestimmten Deckungsgrad in der sozialen Krankenversicherung in Anspruch zu nehmen. Die Analyse weist nach, dass die guten Risiken sich, ohne den Transfer an die schlechten Risiken zu verringern, dadurch besserstellen können, dass sie eine geringere Menge Sozialversicherungsschutz zu einen höheren Preis pro Einheit kaufen.

Ferner konnte gezeigt werden, dass im theoretischen Extremfall eines gleichen Loading-Faktors in der Sozial- und Privatversicherung ein Wettbewerbssystem dazu führt, dass sich für die guten Risiken bzw. hohen Einkommen die Grenzkosten als Funktion des gesamten in Anspruch genommenen Deckungsgrades im Vergleich zu einer reinen Privatversicherungslösung nicht verändern. Das hat zur Konsequenz, dass diese Versichertengruppen unabhängig von ihrer Mitgliedschaft in der Sozialversicherung über ihren gesamten Krankenversicherungsschutz entscheiden und private Zusatzversicherungen nur dann nachfragen, wenn ihr insgesamt gewünschter Versicherungsschutz den in der Sozialversicherung gebotenen Deckungsgrad übersteigt. Bei Lockerung der Annahme eines gleichen Loading-Faktors bei allen Versicherungen sind eine Vielzahl von Kurven, welche die Versicherungsmöglichkeiten wiedergeben, denkbar. Herausgegriffen wurde die Möglichkeit eines geringeren Loading-Faktors in der Sozialversicherung, um eine Erklärung für den in der Praxis nicht fliessend vonstatten gehenden Übergang von der sozialen Grund- zur privaten Zusatzversorgung anzubieten. Die systematische Aufarbeitung aller denkbaren Kostenverläufe (insbesondere auch der nichtlinearen) würde sich schnell sehr stark verästeln. Die hier vertretene Grundaussage aber, dass die Selbstselektion von Versicherten in der sozialen Krankenversicherung effizienzsteigernd eingesetzt werden kann, ohne den Solidarausgleich in Frage zu stellen, bleibt gültig.

\section{Literatur}

Breuer, Michael, 1998: Ökonomische Grundlagen der Sozialversicherungsorganisation. Die Konsequenzen des Groucho-Marx-Effektes. Baden-Baden, Nomos (im Erscheinen).

Buchholz, Wolfgang; Wiegard, Wolfgang, 1992: „Allokative Überlegungen zur Reform der Pflegefürsorge“, Jahrbücher für Nationalökonomie und Statistik 209: 441-457.

Henke, Klaus-Dirk, 1996: „Die Stärkung des Wettbewerbs ist eine sinnvolle Option“, Wirtschaftsdienst 76: 447-449.

Leber, Wulf-Dietrich, 1992: „Aufgaben und Auswirkungen eines Risikoausgleichs bei der Neuordnung des Kassenwettbewerbs in der gesetzlichen Krankenversicherung“, in: Oberender, Peter (Hrsg.): Steuerungsprobleme im Gesundheitswesen. Baden-Baden: Nomos: 169-192. 
Mitchell, Olivia S.; Zeldes, Stephen P., 1995: „Social Security Privatization: A Structure for Analysis“, American Economic Review 86: 363-367.

Rothschild, Michael; Stiglitz, Joseph E., 1976: „Equilibrium in Competitive Insurance Markets: An Essay on the Economics of Imperfect Information, Quarterly Journal of Economics 90: 629-650.

Strassl, Wolfgang, 1988: Externe Effekte auf Versicherungsmärkten. Eine allokationstheoretische Begründung staatlicher Regulierung. Tübingen: Mohr.

van de Ven, Wynand P.M.M., 1992: „How Can We Prevent Cream Skimming in a Competitive Health Insurance Market?“, in: Zweifel, Peter; Frech III, H.E. (eds.): Health Economics Worldwide. Dodrecht, Boston, London: Kluwer: 23-46.

Wilson, C., 1977: „A Model of Insurance Markets with Incomplete Information“, Journal of Economic Theory 12: 167-212.

Windisch, Rupert, 1998: „Gleichgewichtskonzepte für wettbewerbliche Versicherungsmärkte bei asymmetrischer Information“, WISU 8-9/98: 935-943. 